A novel on-field training intervention improves novice goalkeeper penalty

\title{
kick performance
}

1: Department of Sport and Exercise Science, University of Portsmouth, UK

2: Expert Performance and Skill Acquisition Research Group, School of Sport, Health and Applied Science, St Mary's University, Twickenham, UK

3: MOVE Research Institute Amsterdam, Faculty of Behavioral and Movement Sciences, Vrije Universiteit Amsterdam, The Netherlands 4: Institute of Human Performance, University of Hong Kong, Hong Kong SAR

\section{Corresponding author:}

Dr Matt Dicks, Department of Sport and Exercise Science, University of Portsmouth, UK

E-mail: matt.dicks@port.ac.uk

As accepted for publication in The Sport Psychologist on $22^{\text {nd }}$ September, 2016 doi:10.1123/tsp.2015-0148 
ON-FIELD PERCEPTUAL TRAINING 
Abstract

This study developed an on-field anticipation training intervention with the aim of improving novice goalkeeper penalty kick performance. Eighteen participants were allocated to either one-player (OP); or three-player (TP) training. The OP group faced "traditional" practice, with one player running-up to execute each kick. The TP group faced three players in a form of variable practice; two players stopped their run-up approximately $1.2 \mathrm{~m}$ from the ball with the third-player executing the kick. Following training, results revealed that TP made significantly more saves when facing non-deception kicks in comparison with OP. An implication for applied practice is that there are potential gains to be made through training anticipation skills via new on-field practices rather than the current research focus on video-based training. 


\section{Introduction}

Research in the visual anticipation literature has revealed that differences in the performance accuracies of expert and less-skilled performers can be reconciled by variations in the locations of information pick-up and timing of actions (Triolet, Benguigui, Le Runigo, \& Williams, 2013). For instance, when anticipating the direction of an opponent's deceptive movements, elite rugby players outperformed novices by attending to honest (centre of mass) information and waiting later before initiating their movement response (Brault, Bideau, Kulpa \& Craig, 2012). In contrast, the earlier response time of novices did not negatively affect performance for non-deceptive movements, where both experts and novices achieved ceiling levels of anticipation accuracy (97\%) (see also, Jackson, Warren \& Abernethy, 2006). These findings are corroborated by football penalty kick research, where results indicate that penalty takers' use of deception ensures that early kinematic information (e.g., approach angle) is incongruent with kick direction (Lopes, Jacobs, Travieso \& Araújo, 2014). However, if goalkeepers attend to kinematic information (e.g., non-kicking foot placement) that unfolds when the penalty taker is approximately $1.2 \mathrm{~m}$ from the ball, this increases the likelihood of success when facing deceptive kicks (Dicks, Button, \& Davids, 2010).

Differences in anticipation between expert and less-skilled performers, have led perceptual learning researchers to explore the benefits of different training methods (see Farrow, 2013, for a review). Recently, perspectives in ecological psychology have proposed that variability in practice conditions may be particularly effective in improving anticipation accuracy (see Dicks, van der Kamp, Withagen \& Koedijker, 2015; Smeeton, Huys \& Jacobs, 2013). For example, Smeeton and colleagues (2013) revealed that the prediction of tennis serve direction can be improved through the implementation of reduced usefulness training, which has the aim of directing novice (learners) search to more reliable information through changes in practice conditions. It is thought that variable practice conditions reduce the availability of variable or less useful information (e.g., early run-up information from a penalty taker), while 
information with minimal variability (e.g., the orientation of the penalty taker's non-kicking foot) (Dicks, Button et al., 2010; Lopes et al., 2014) remains present. Thus, variable practice which leads to a reduction in the availability of less useful (variant) information is thought to force learners to search for alternative, more reliable information (Smeeton et al., 2013).

The aim of this study was to examine whether a novel on-field training intervention improves the anticipation performance of novice football goalkeepers for deceptive and nondeceptive penalty kicks. Participants were allocated to either a one-player training group (OP) or a three-player training group (TP). The OP group faced "traditional" practice, with one penalty taker running up to execute the kicks. The TP group faced three players running-up to the ball, with only one of the three players continuing the run-up to execute the kick. The other two players stopped their run-up approximately 1.2m from the ball (cf. Dicks, Button et al., 2010). The rationale for choosing this distance, and subsequently the aim of TP, was to orient goalkeeper attention towards the more reliable information that unfolds towards the end of the run-up. Following Smeeton et al. (2013), we rationalised that TP would act as a form of reduced usefulness training, by minimising the availability of early penalty taker information. If TP achieves this aim, we hypothesised that the TP group would learn to attend to reliable information in the kicking action of penalty takers (e.g., non-kicking foot placement) and perform significantly better than OP, leading to better post-test anticipation performance in deception and non-deception trials.

\section{Method}

\section{Participants}

Eighteen novice goalkeepers (Mage $=20.89 \pm 0.96$ years $)$ participated in the study. All participants were male and had at least three years' football playing experience at a competitive recreational level but no specific experience as a goalkeeper. Five penalty takers (Mage $=21.17$ \pm 0.98 years) were recruited to execute penalty kicks. All penalty takers had between five and 
ten years' experience at a competitive recreational level and experience of taking penalties in competition. The players had no previous experience of taking penalties against any of the goalkeepers. Ethical approval was obtained from the local University ethics committee and all participants provided written informed consent.

\section{Apparatus and Procedure}

The pre-test and post-test, consisted of 30 penalty kicks executed by three different players. All participants faced kicks from the same three players in the pre-test and post-test. The penalty takers approached the ball from a distance of $4 \mathrm{~m}$ and followed a script that determined the angle of run-up to the ball, which side of the goal to aim for (bottom left, bottom right) and whether to use deception or no deception (see Dicks, Button et al., 2010). During deception trials, players executed kicks as though they intended to aim to one side of the goal, before shooting at the opposite side. In non-deception trials, the penalty taker shot directly at the desired goal location without any deceptive intent (Lopes et al., 2014). The script ensured that the three penalty takers executed 10 penalties each (five deception, five non-deception) that were directed evenly to the bottom corners of the goal. In addition to the 30 penalties, a further six kicks were executed to various predetermined goal locations to remove participants' awareness of the task procedure (cf. Dicks, Button et al., 2010). Each player took two of these kicks and goalkeeping performance was not analysed for these trials. All penalty kicks were executed at a full size goal $(7.32 \times 2.44 \mathrm{~m})$, using a size five football from the regulation distance $(11 \mathrm{~m})$ on an outdoor Astroturf pitch.

Training. Following the pre-test, performances were ranked based on the number of saves for deception and non-deception trials, before allocating participants to one of two training groups in order to ensure an equal range of visual anticipation performance at baseline for the two groups (cf. Hopwood, Mann, Farrow, \& Nielsen, 2011). The two training groups were as follows: one-player training (OP) and three-player training (TP). Participants in both groups faced a total of 80 kicks distributed equally across four training sessions during the 
intervention (Smeeton, Williams, Hodges \& Ward, 2005). The OP training consisted of "traditional" kicks in which one player ran-up from a distance of $4 \mathrm{~m}$ and executed the penalty.

The TP training consisted of three players running up to the ball from $4 \mathrm{~m}$, side-by-side, at three different orientations to the ball (left, central, right), with only one of the three players executing the penalty. The ordering of when each of the three players executed the kick was randomised. The other two penalty takers stopped their run-up $1.2 \mathrm{~m}$ from the ball (Dicks, Button et al., 2010). It was pre-arranged which player was going to take each penalty although goalkeepers were not aware of this arrangement. Different markers were placed along the approach to the ball, and unknown to the goalkeepers, one pair of markers denoted $1.2 \mathrm{~m}$ from the ball. Penalty takers in TP and OP training did not follow a script but checks were made in order to ensure an even distribution of kicks to either side of the goal.

\section{Dependent Measures and Analysis}

Goalkeeper performance for deception and non-deception trials was assessed by recording the number of dives to the correct side of the goal and the number of saves in each condition. Tests of normality indicated the data to be normally distributed. For dives, one sample t-tests were performed on post-test performance to determine if training led to performance that was greater than chance. Number of saves were analysed using a two (group: OP, TP) x two (testing phase: pre-test, post-test) analysis of variance (ANOVA). Pre-test performance was analysed using an independent samples t-test to ensure there were no differences between the OP and TP group prior to training. Effect sizes are reported using $\eta^{2}$ for ANOVA and Cohen's $d$ for post-test comparisons.

\section{Results}

For the TP group, the number of dives to the correct side of the goal was statistically greater than chance (7.5) for both deception $(M=10.33, S D=2.06)$ and non-deception $(M=$ $10.78, S D=2.86), t \mathrm{~s}(8)=4.12$ and 3.44 , respectively, $p s<.01$. In contrast, for the OP training 
group, there was no difference between the number of dives to the correct side of the goal and chance for both deception $(M=9.11, \mathrm{SD}=2.67)$ and non-deception $(\mathrm{M}=8.33, \mathrm{SD}=1.66)$, $t \mathrm{~s}(8)=1.81$ and 1.51 , respectively, $p s>.05$

Independent samples t-test revealed no differences in the pre-test between the OP and TP groups for number of saves in non-deception, $t(16)=.263, p=.796, d=0$, and deception trials $t(16)=-.447, p=.661, d=0.12$. The subsequent ANOVA showed for non-deception trials that there was a significant main effect for group, $F(1,16)=6.682, p<.05, \eta^{2}=0.29$, testing phase, $F(1,16)=11.22, p<.01, \eta^{2}=0.41$, and a significant interaction effect, $F(1,16)=14.01$, $p<.01, \eta^{2}=0.47$ (Figure 1). Follow-up tests revealed the TP training group made significantly more saves than the OP training group in the post test for non-deception trials, $t(16)=-4.03, p<$ $.001, d=-1.89$. The TP training group made significantly more saves in the post-test compared to the pre-test, $t(8)=-5.37, p<.005, d=-2.01$, but no significant difference was found between the two testing phases for the OP training group, $t(8)=0.26, p=0.79, d=0.11$.

\section{Insert Figure 1 Here}

For deception trials, there was no significant main effect for group $F(1,16)=0.045, p=$ $0.83, \eta^{2}=0.003$, testing phase, $F(1,16)=0.15, p=0.70, \eta^{2}=0.009$, and there was no significant interaction effect, $F(1,16)=0.15, p=0.70, \eta^{2}=0.009$ (Figure 2).

\section{Insert Figure 2 Here}

\section{Discussion}

The present study examined whether a highly feasible, new on-field training intervention improved novice goalkeeper penalty kick performance. The aim of TP, as a form of reduced usefulness training (Smeeton et al., 2013), was to direct goalkeeper attention towards the use of kinematic information that unfolds in the final phase of a penalty taker's kicking action (Dicks, Button et al., 2010). For dives to the correct side of the goal, results revealed that the TP group performed significantly better than chance in the post-test for both deception and 
non-deception trials. In contrast, there was no difference from chance for the OP group for both kick conditions. Moreover, results revealed that the TP group made significantly more saves than OP during non-deception trials following training (Figure 1). There were no differences in the number of saves between the two groups for deception trials (Figure 2).

For non-deception, the TP group performed above chance levels following training and showed a significant improvement for number of kicks saved in comparison with the OP group. Thus, comparable to previous video-training research, the intervention used in the present study indicates the potential benefit of using variable practice conditions for enhancing anticipation performance (Dicks et al., 2015; Smeeton et al., 2013). Further to previous video-training studies (e.g., Hopwood et al., 2011), our approach has shown that performance improvements can be elicited from research-informed modifications to existing on-field training interventions. The implication of this finding for applied practitioners is that new on-field training practices are a viable intervention to enhance athlete anticipation and decision-making skills (Ford, Yates, \& Williams, 2010). In this respect, there are potential gains to be made by exploring new approaches aimed at training anticipation skill via variations of on-field practice rather than the current research focus of video-based training (Dicks et al., 2015).

For deception, the TP group performed above chance levels following training although the TP group did not make more saves in comparison with the OP group. Previous research has suggested that the timing of actions in anticipation tasks influences performance accuracy (Triolet et al., 2013). Specifically, in the case of anticipating deceptive actions, moving early can lead to an increased likelihood of being deceived (Brault et al., 2012). Furthermore, penalty kick findings indicate that the timing of movement initiation is correlated to goalkeeper agility, with slower goalkeeper's moving earlier and having an increased susceptibility to deception (Dicks, Davids \& Button, 2010). It is therefore plausible that the differences observed for the effects of training in response accuracies for deception and non-deception kicks could be reconciled by the timing of goalkeeper movements. However, as movement times were not 
recorded, we are unable to verify this claim. Given the complexity of anticipation tasks, which comprise deception, it is possible that novices may benefit less from the mode of reduced usefulness training examined in this study in comparison with more skilled goalkeepers. Indeed, the short period of 80 practice trials (cf. Smeeton et al., 2005) used in the current study may be insufficient for eliciting meaningful improvements in real-time anticipation skill, particularly given that no differences were observed in the OP group from pre- to post-test. Future work, including gaze control and movement measures, participants of different skill levels, and interventions of different durations is needed to understand the changes in control mechanisms that occur after a period of perceptual training (Dicks et al., 2015).

In conclusion, the present study had the aim of training novice goalkeepers to exploit the kinematic information that unfolds in the penalty taker's kicking action (i.e., approximately the final $1.2 \mathrm{~m}$ of the penalty taker's run-up). Following training, goalkeepers in the TP group performed above chance levels for both deception and non-deception trials but this was not the case for OP. When facing non-deception kicks, goalkeepers in the TP group made significantly more saves in comparison with OP. In contrast, novice goalkeepers in the TP group did not make more saves than OP in deception trials. Future research is needed to build on these efforts through the implementation of rigorous measures (e.g., gaze and movement control) to fully capture changes in control processes following training. An implication for applied practitioners is that there are potential gains to be made through new approaches to training anticipation skills via on-field practice rather than the current focus on video-based training (Dicks et al., 2015).

\section{Acknowledgements}

The authors would like to acknowledge the hard-work and contributions of John Burkitt, Jack Houghton, Liam Scofield, Jasdeep Sandhu, and Tim Locke during the participant recruitment and testing phases of this experiment. 
Brault, S., Bideau, B., Kulpa, R., \& Craig, C. M. (2012). Detecting deception in movement: the case of the side-step in rugby. PLoS ONE, 7(6), e37494. doi:10.1371/journal.pone.0037494

Dicks, M., Button, C., \& Davids, K. (2010). Availability of advance visual information constrains association-football goalkeeping performance during penalty kicks. Perception, 39(8), 1111-1124. doi:10.1068/p6442

Dicks, M., Davids, K., \& Button, C. (2010). Individual differences in the visual control of intercepting a penalty kick in association football. Human Movement Science, 29(3), 401-411. doi:10.1016/j.humov.2010.02.008

Dicks, M., van der Kamp, J., Withagen, R., \& Koedijker, J. (2015). “Can we hasten expertise by video simulations?" Considerations from an ecological psychology perspective. International Journal of Sport Psychology, 46, 109-129. doi:10.7352/IJSP2015.46.

Farrow, D. (2013). Practice-enhancing technology: a review of perceptual training applications in sport. Sports Technology, 6(4), 170-176. doi:10.1080/19346182.2013.875031

Ford, P., Yates, I., \& Williams, A. M. (2010). An analysis of practice activities and instructional behaviours used by youth soccer coaches during practice: exploring the link between science and application. Journal of Sports Sciences, 28(5), 483-495. doi:10.1080/02640410903582750

Hopwood, M. J., Mann, D. L., Farrow, D., \& Nielsen, T. (2011). Does visual-perceptual training augment fielding performance of skilled cricketers? International Journal of Sports Science \& Coaching, 6(4), 523 - 535. doi:10.1260/1747-9541.6.4.523

Jackson, R. C., Warren, S., \& Abernethy, B. (2006). Anticipation skill and susceptibility to deceptive movement. Acta Psychologica, 123(3), 355-371. doi:10.1016/j.actpsy.2006.02.002 
Lopes, J. E., Jacobs, D. M., Travieso, D., \& Araújo, D. (2014). Predicting the lateral direction of deceptive and non-deceptive penalty kicks in football from the kinematics of the kicker. Human Movement Science, 36, 199-216. doi:10.1016/j.humov.2014.04.004

Smeeton, N.J., Huys, R., \& Jacobs, D. M. (2013) When less is more: reduced usefulness training for the learning of anticipation skill in tennis. PLoS ONE 8(11): e79811. doi:10.1371/journal.pone.0079811

Smeeton, N. J., Williams, A. M., Hodges, N. J., \& Ward, P. (2005). The relative effectiveness of various instructional approaches in developing anticipation skill. Journal of Experimental Psychology: Applied, 11(2), 98-110. doi:10.1037/1076-898X.11.2.98

Triolet, C., Benguigui, N., Le Runigo, C., \& Williams, A. M. (2013). Quantifying the nature of anticipation in professional tennis. Journal of Sports Sciences, 31(8), 820-830. doi:10.1080/02640414.2012.759658 
260

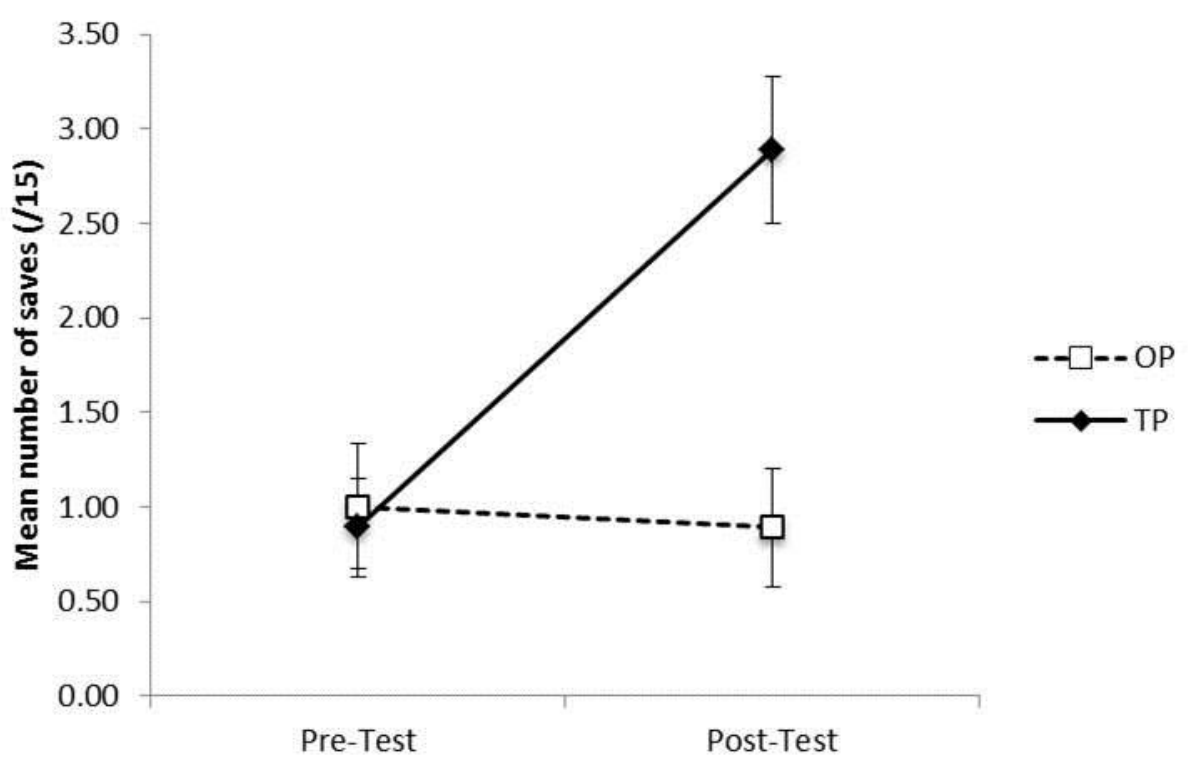

262 Figure 1: Mean number of saves in response to non-deception kicks for one-player (OP) and three-player (TP) training groups. 


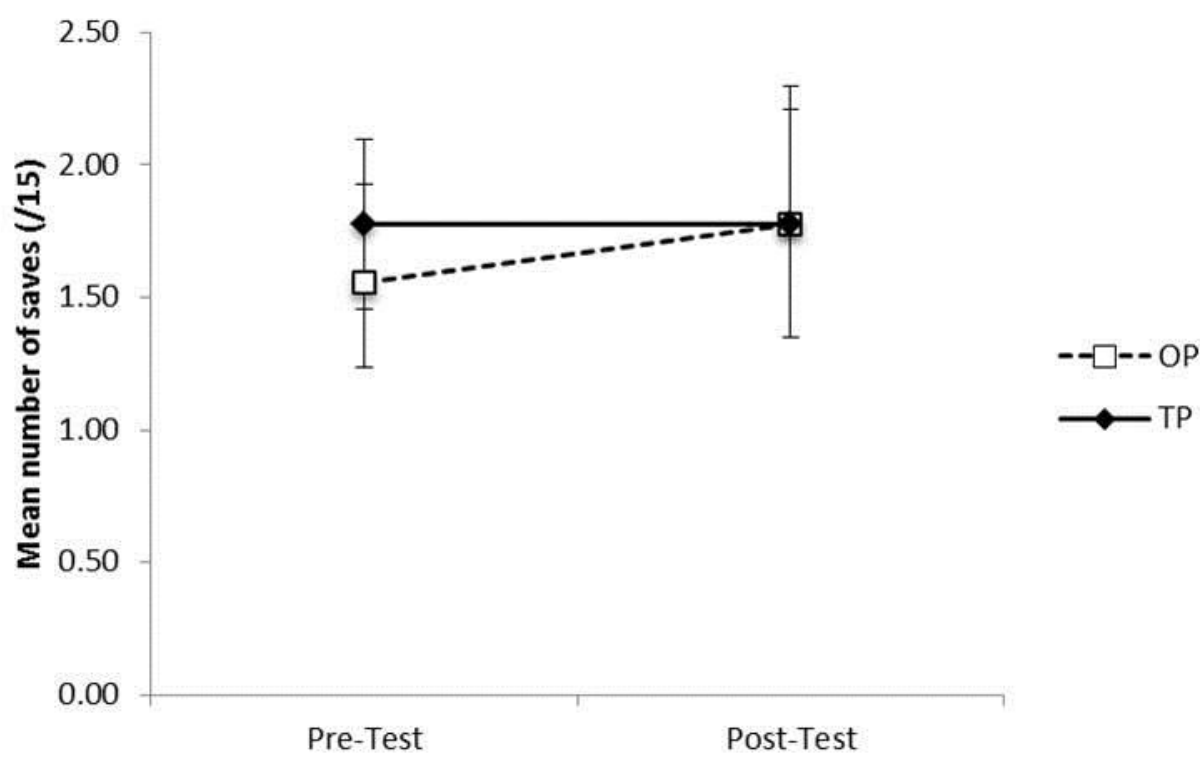

266 Figure 2: Mean number of saves in response to deception kicks for one-player (OP) and threeplayer (TP) training groups. 